\title{
Antisense Knockdown of Kras Inhibits Fibrosis in a Rat Model of Unilateral Ureteric Obstruction
}

\author{
Jia-Hui Wang, ${ }^{*}$ Lucy J. Newbury, ${ }^{*}$ A.S. Knisely, ${ }^{\dagger}$ \\ Brett Monia, ${ }^{\ddagger}$ Bruce M. Hendry, ${ }^{*}$ and \\ Claire C. Sharpe* \\ From the Department of Renal Medicine* and the Institute of \\ Liver Studies, ${ }^{\dagger}$ King's College Hospital, London, United Kingdom; \\ and ISIS Pharmaceuticals, ${ }^{\ddagger}$ Carlsbad, California
}

Tubulointerstitial fibrosis is the hallmark of chronic kidney disease and is characterized by an increase in the number and activity of interstitial fibroblasts and by excessive matrix deposition. Ras is an intracellular signaling molecule involved in cell proliferation and differentiation. It has recently been implicated in the pathogenesis of renal fibrosis. Of the three different isoforms of Ras (Kirsten, Harvey, and Neural), we previously demonstrated that the Kirsten isoform is key in the control of renal fibroblast proliferation in vitro. In this study, we used gene therapy in the form of antisense oligonucleotides (ASOs) specifically to silence Kras (alias Ki-ras) expression in a rat model of renal fibrosis caused by unilateral ureteric obstruction. We demonstrate that renal Kras expression increases by $\mathbf{7 0} \%$ in this model compared with shamoperated animals and that treatment with ASOs can reduce total renal Kras by $>90 \%$ to levels well below basal. This silencing is associated with a dramatic inhibition of interstitial fibrosis, a fivefold reduction in $\alpha$-smooth muscle actin expression, and a 2.4 -fold reduction in collagen I deposition. This inhibition was observed despite histologic evidence of marked interstitial inflammation. These findings demonstrate that silencing Kras expression can markedly inhibit renal fibrosis. This strategy should be considered as a new potential therapeutic avenue. (Am J Pathol 2012, 180: 82-90; DOI: 10.1016/j.ajpath.2011.09.036)

Chronic kidney disease is a large and expanding problem worldwide. Whatever the initiating insult, the presence of progressive chronic kidney disease is associated with scarring of the tubulointerstitial compartment. Interstitial myofibroblasts are responsible for the laying down of extracellular matrix, ${ }^{1}$ and although sparse in the normal kidney, after injury they increase in number via several potential mechanisms, such as proliferation, pericyte and fibrocyte differentiation and migration, and epithelial to mesenchymal transition. ${ }^{2,3} \mathrm{Al}-$ though complex, the many fibrogenic cell signals and phases of fibrosis are clearly described. ${ }^{4}$ Despite this large body of knowledge, there is still no effective, fibrosis-specific therapeutic agent that has been adopted into clinical practice.

Ras monomeric GTPases are transduction molecules in many extracellular signaling pathways that regulate cell proliferation, survival, and differentiation. ${ }^{5}$ Although Ras family species are, to date, of particular interest in oncology, ${ }^{6}$ we and others have accumulated a substantial amount of evidence for a role for the Ras monomeric GTPases in the pathogenesis of renal disease and in epithelial to mesenchymal transition. Ras family members are downstream convergence points for signal cascades mediated by many cell surface receptors with ligands expressed in renal injury, such as transforming growth factor $\beta$, platelet-derived growth factor, angiotensin II, and epidermal growth factor, allowing transduction of signal to downstream profibrotic effectors. As such, they may be an important potential target for treating the fibrosis of chronic kidney disease. ${ }^{7-14}$

Recently, Bechtel et $\mathrm{al}^{15}$ demonstrated a direct link between Ras activation and renal fibrogenesis. They showed that hypermethylation of RASAL1 in fibrotic kidneys results in decreased expression of the Ras inhibitory

Supported in part by the National Institute for Health Research, UK (clinician scientist award DHCS/G121/33 to C.C.S.), and Kidney Research UK (project grant RP37/1/05 to C.C.S. and clinical training fellowship TF10/2006 to J.-H.W.). ISIS Pharmaceuticals, which designs and develops antisense oligonucleotides as potential therapeutic agents, provided the oligonucleotides used in this study free of charge.

Accepted for publication September 26, 2011.

Disclosure: B.M. is an employee of ISIS Pharmaceuticals.

Supplemental material for this article can be found at http://ajp. amjpathol.org or at doi: 10.1016/j.ajpath.2011.09.036.

Address reprint requests to Claire C. Sharpe, F.R.C.P., Ph.D., Department of Renal Medicine, King's College London, 10 Cutcombe Rd., London SE5 9RJ, UK. E-mail: Claire.sharpe@kcl.ac.uk. 
protein RASAL1. This correlates with an increase in Ras activity and a subsequent increase in fibrosis in several murine models of renal fibrosis. The group concluded that hyperactive Ras contributes to fibroblast activation and fibrosis and that the antifibrotic capacity of Ras inhibitors should be further explored.

There are three separate isoforms of Ras: Kirsten (Kras; alias Ki-Ras), Harvey (Hras; alias Ha-Ras), and Neural (Nras; alias N-Ras). Although the isoforms are 85\% homologous, they differ in their C-terminal 24 amino acids, a "hypervariable region" of the molecule. This region confers on each isoform distinct characteristics with respect to cell localization and function. ${ }^{16,17}$ Our group previously demonstrated that Kras is the predominantly expressed isoform in human renal fibroblasts and that knockdown of this isoform by antisense oligonucleotides (ASOs) results in significant inhibition of fibroblast proliferation. ${ }^{18,19}$ In this study, we investigated whether silencing of Kras (alias Ki-ras) expression using ASOs is antifibrotic in a rat model of unilateral ureteric obstruction (UUO).

\section{Materials and Methods}

\section{Oligonucleotides}

ASOs were designed and produced by ISIS Pharmaceuticals, Carlsbad, California. They are 20-nucleotide, fully phosphorothioate "gapmers." The five sugar residues on each end are 2'-O-methoxy-ethyl modified. The central 10 sugars are deoxyribonucleotides. All pyrimidine bases are 5-methyl substituted (T and me5-C). The oligonucleotide sequences were as follows: ASO 1, 5'-ATTCACATGACTATACACCT-3'; ASO 2, 5'-CACACTTATTCCCTACTAGG-3'; SO 1, 5'-ATTAACACGCCTACATACTT-3'; SO 2, 5'-CCCACTAAATCCGTTCTATG-3'; CO 1, 5'-TCAGTAATAGCCCCACATGG-3'; and CO 2, 5'-CCTTCCCTGAAGGTTCCTCC-3' . CO 1 was used for in vitro experiments because this was the control used in a previous in vitro study. ${ }^{18} \mathrm{CO} 2$ is a standard control oligonucleotide used by ISIS Pharmaceuticals for in vivo work and was used for the initial in vivo experiments. SO 1 and SO 2 were subsequently designed for this project and are scrambles of ASO 1 and ASO 2, respectively.

\section{Primary Culture and Cell Lines}

Primary rat fibroblasts were cultured as previously described. ${ }^{18}$ Briefly, rat kidneys were decapsulated in sterile ice-cold HBSS. Cortices were removed and fragmented under sterile conditions. This tissue was digested in a $1-\mathrm{mg} / \mathrm{mL}$ solution of collagenase type IV (SigmaAldrich Co. Ltd., Dorset, UK) and incubated in a water bath at $37^{\circ} \mathrm{C}$ for 30 minutes. The digestion process was terminated by the addition of ice-cold $2 \%$ fetal calf serum (Sigma-Aldrich Co. Ltd.)/HBSS solution. The digest was then passed through a $100-\mu \mathrm{m}$ sieve, and the filtrate was centrifuged at $200 \times g$ for 5 minutes at $4^{\circ} \mathrm{C}$ to pellet cellular material. This material was then washed in HBSS and resuspended in $30 \mathrm{~mL}$ of a $50 \%$ Percoll solution (Sigma-Aldrich Co. Ltd.) and centrifuged at $4^{\circ} \mathrm{C}$ at $30,000 \times g$ for 30 minutes. The top band in the gradient obtained contained the fibroblast fraction, which was harvested and cultured in Dulbecco's modified Eagle's medium Ham's F-12 (Gibco BRL, Life Technologies, Paisley, UK) supplemented with $10 \%$ fetal calf serum.

Normal adult rat fibroblast cells (NRK-49F) were obtained from the European Collection of Cell Cultures (6101301) and cultured in Dulbecco's modified Eagle's medium supplemented with penicillin $(100 \mathrm{IU} / \mathrm{mL})$, streptomycin $(100 \mu \mathrm{g} / \mathrm{mL})$, amphotericin $(2.5 \mu \mathrm{g} / \mathrm{mL})$, and $10 \%$ fetal calf serum.

\section{Semiquantitative $R T$-PCR}

Total cellular RNA was extracted using a Qiagen RNeasy minikit (Qiagen, Crawley, UK). Promega's Access RTPCR kit (Promega, Southampton, UK) was used for semiquantitative RT-PCR analysis of sample RNA. The primer pairs targeting Kras and $\beta$-actin in the rat had the following forward and reverse sequences, respectively: Kras, 5'-AGAGTGCCTTGACGATACAGC-3' and 5'-TCCCTCATTGCACTGTACTCC-3'; $\beta$-actin, 5'-CCCACACTGTGCCCATC-3' and 5'-TGATCCACATCTGCTGGA-3'. Samples were amplified for 22 cycles of PCR, and the products were separated on a $1.5 \%$ agarose gel.

\section{In Vitro Transfection of Oligonucleotides}

Oligonucleotides were diluted with Opti-MEM (Gibco $\mathrm{BRL}$ ) to form a $10-\mu \mathrm{mol} / \mathrm{L}$ stock. Cells were grown to a confluence of $50 \%$ to $70 \%$ and were washed with PBS before transfection. Oligonucleotides were transfected into cells using Lipofectin (Gibco BRL), a cationic liposomal system. Lipofectin, $1.25 \mu \mathrm{L}$, was used per 100 $\mathrm{nmol} / \mathrm{L}$ oligonucleotide per $100 \mu \mathrm{L}$ of final transfection volume. The final concentration of oligonucleotides used for transfection was $200 \mathrm{nmol} / \mathrm{L}$. Cells were transfected for 6 to 12 hours before the transfection medium was removed and replaced with normal culture medium.

\section{UUO Model}

All the experimental procedures were approved under provisions of the Animals (Scientific Procedures) Act 1986 and were performed under license number PPL 70/6054. UUO was caused in adult male Wistar rats. After a midline incision was made, the left ureter was isolated and ligated with two 6/0 silk ties. The abdomen was closed, and buprenorphine $(30 \mu \mathrm{g} / \mathrm{kg})$ was administered subcutaneously. Any first dose of treatment (oligonucleotide or vehicle) was also administered subcutaneously at this point. Animals undergoing the sham procedure underwent a full laparotomy and manipulation of the ureter without ligation. All the animals were sacrificed on day 16 after UUO.

\section{Administration of Oligonucleotides in Vivo}

ASOs and control oligonucleotides were administered by subcutaneous injection in either the nape of the neck or a 
rear limb. Variation of injection points prevented cumulative tissue damage. All oligonucleotides were dissolved in sterile water and administered on alternate days at a dose of $12.5 \mathrm{mg} / \mathrm{kg}$.

\section{RNA Retrieval from Tissue}

RNA was retrieved from tissue using the Qiagen RNeasy minikit. The sample (30 mg) was initially homogenized in $600 \mu \mathrm{L}$ of guanidine-thiocyanate lysis buffer (supplied) using a potter homogenizer. This suspension was then transferred to a QIAshredder (Qiagen), and the manufacturer's protocol was followed. Isolated RNA was quantified, and its purity was established using absorbance spectroscopy.

\section{Quantitative PCR}

Reverse transcription of total RNA was performed using the Omniscript reverse transcription kit (Qiagen) according to the manufacturer's protocol. The TaqMan sequence detection system (Applied Biosystems, Carlsbad, CA) was used, and quantitative PCR (qPCR) was performed using the ABI Prism 7900HT machine (Applied Biosystems). Abundance of target-gene transcript was quantified relative to the endogenous control glyceraldehyde-3-phosphate dehydrogenase. Target and endogenous control levels for each sample were measured in triplicate. TaqMan gene expression assays and TaqMan master mix were purchased from Applied Biosystems.

\section{Histologic Analysis}

Tissue was fixed in buffered formalin for 24 hours, embedded in paraffin, cut into $4-\mu \mathrm{m}$-thick sections, and picked up onto glass slides (plain or polylysine coated). Sections were stained with H\&E.

\section{Special Stains}

Sections stained using the picro-Mallory trichrome (PMT) and picrosirius red (PSR) techniques were used to assess fibrosis. PSR-stained sections were viewed under cross-polarized light and under bright light. The degree of cortical fibrosis was scored by a consultant renal histopathologist in a randomized and blinded manner.

\section{Immunostaining}

Sections mounted on polylysine-coated slides were deparaffinized after baking at $40^{\circ} \mathrm{C}$ overnight. Antigen retrieval was performed by pressure cooking in citrate buffer ( $\mathrm{pH} 6)$ as required. Antibodies used were directed against aquaporin 1 (AB2219; Millipore, Billerica, MA), aquaporin 2 (AB3066; Millipore), modified oligonucleotides (supplied by ISIS Pharmaceuticals, rabbit polyclonal), $\alpha$-smooth muscle actin ( $\alpha$-SMA) (A2547; SigmaAldrich Co. Ltd.), collagen I (AB755P; Millipore), Ki-67 (NCL-L-KI67-MM1; Novocastra, Newcastle on Tyne, UK), ED-1 (MCA341R; Serotec, Kidlington, UK), and FSP-1
(S100A4) (A5114; Dako, Ely, UK). Detection was achieved by a peroxidase substrate kit using a synthetic dextran backbone polymer and diaminobenzidine [Dako EnVision detection system (K5007), Dako]. Sections were assessed after hematoxylin counterstaining. For $\alpha$-SMA and ED-1, slides were scored by measurement of staining intensity and area using Nikon NIS-Elements (basic research) software (Nikon, London, UK). Slides were scored in a blinded manner based on five random cortical views per section at $\times 200$ magnification. Staining for collagen 1 was scored by a consultant renal histopathologist in a randomized and blinded manner. Staining for Ki-67 and FSP-1 was scored in a blinded manner using light microscopy at $\times 200$ magnification. Ten random fields of cortex were evaluated, with assignment of cells to glomerular, tubular, or interstitial compartments.

\section{Western Blot Analysis}

Western blot analysis was performed using antibodies against $\alpha$-SMA (A2547; Sigma-Aldrich Co. Ltd.) and panRas (OP40; Merck, Darmstadt, Germany). Glyceraldehyde-3-phosphate dehydrogenase (MAB374; Millipore) was used as a loading control for $\alpha$-SMA blots.

\section{Immunoprecipitation}

Due to a relatively low abundance of Ras within the cell, Ras protein was concentrated from crude protein lysates using immunoprecipitation; $0.5 \mathrm{mg}$ of protein was used. All the volumes were normalized to $1 \mathrm{~mL}$ with PBSTDS [1× PBS, $1 \%$ Triton X-100 (Roche Diagnostics GmbH, Mannheim, Germany), $0.5 \%$ sodium deoxycholate, $0.1 \%$ SDS, $0.5 \mu \mathrm{g} / \mathrm{mL}$ of leupeptin, $1.0 \mu \mathrm{g} / \mathrm{mL}$ of pepstatin, 1.0 $\mathrm{mmol} / \mathrm{L}$ EDTA, and phenylmethylsulfonyl fluoride 0.2 $\mathrm{mmol} / \mathrm{L}$ ]. Anti-Ras-agarose conjugate, $15 \mu \mathrm{L}$ (OP01A; Merck), was added per milliliter volume, and all the samples were incubated, under rotation, at $4^{\circ} \mathrm{C}$ overnight. Samples were then washed and separated by SDSPAGE for analysis.

\section{Statistical Analysis}

Statistical analysis of data was performed using GraphPad Prism version 4 software (GraphPad Software Inc., La Jolla, CA). When comparing two sets of data, an unpaired Student's t-test was used. For group data, analysis of variance testing was initially performed. Bonferroni adjustment was then used to correct for multiple testing.

\section{Results}

\section{ASOs Reduce Kras Expression in Vitro}

ASOs consist of single strands of 20-22mer oligonucleotides that are complementary to their target mRNA sequence. Binding of ASOs to target induces RNase $\mathrm{H}$ and subsequent target mRNA hydrolysis, thereby effecting "gene silencing." Oligonucleotides administered systemically are filtered at the glomerulus and are rapidly taken 
A

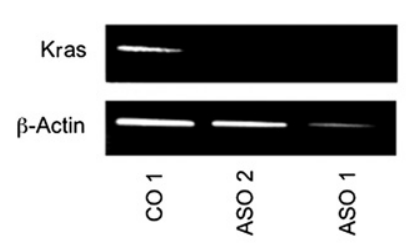

C

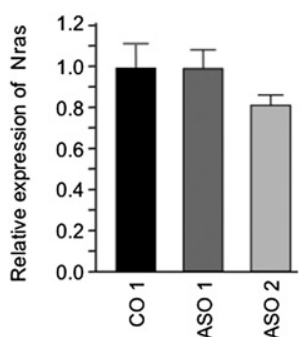

B
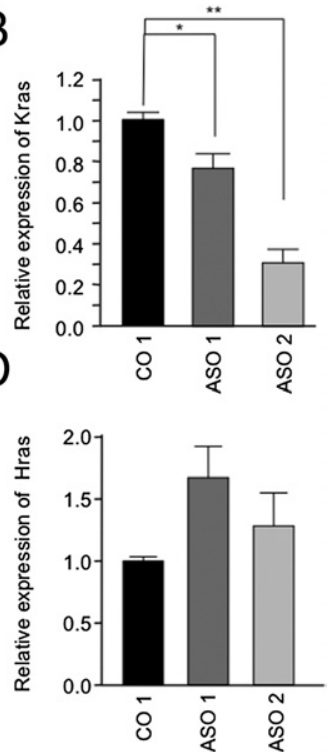

Figure 1. ASOs reduce Kras expression in vitro. A: Primary culture rat renal fibroblasts were transfected with $200 \mathrm{nmol} / \mathrm{L}$ control oligonucleotide (CO 2) or antisense oligonucleotides (ASO 1 and ASO 2). After 24 hours, the cells were lysed, and extracted mRNA was subjected to semiquantitative RT-PCR for Kras. $\beta$-actin primers were used to detect this housekeeping gene. B-D: NRK-49F cells were transfected with $200 \mathrm{nmol} / \mathrm{L}$ control oligonucleotide (CO 2 ) or antisense oligonucleotides (ASO 1 and ASO 2). After 24 hours, the cells were lysed, and extracted mRNA was subjected to RT-qPCR for Kras, Nras, and Hras as indicated. $n=3$ separate experiments. Error bars are SEM. * $P$ $<0.05,{ }^{* * *} P<0.001$

up by tubular epithelial cells. Thus, they are an attractive therapeutic option when considering potential therapies for renal disease. ${ }^{20}$

Oligonucleotides ISIS 104440 (ASO 1) and ISIS 104419 (ASO 2) target the Kirsten isoform of Ras in the rat. Four separate control oligonucleotides were used in this study. All the oligonucleotides, control and antisense, were 20-mer in length. Control oligonucleotide 15167 (CO 1) targets the HIV promoter sequence but has no recognized target in the rat. Control oligonucleotide 141923 (CO 2) also has no recognized target in the rat. Control oligonucleotides 417230 (SO 1) and 417226 (SO 2) are scrambled sequences of ASO 1 and ASO 2, respectively.

Oligonucleotides were initially evaluated for specificity and efficacy in vitro using primary rat renal fibroblasts in culture. After transfection, RNA was extracted, and semiquantitative PCR was used to demonstrate knockdown of Kras expression by transfection with ASO 1 and ASO 2 compared with CO 1 (Figure 1A). For further validation, normal rat kidney cells [NRK-49F, European Collection of Cell Cultures (86101301)] were transfected with ASO 1 and ASO 2. Both oligonucleotides significantly reduced expression of Kras mRNA by $23 \%$ and $69 \%$, respectively, compared with $\mathrm{CO} 1$ as assessed by RT-qPCR, with no significant knockdown of either the Nras or Hras isoforms (Figure 1, B-D). However, a nonsignificant trend toward up-regulation of the latter isoform was noted, with an increase in expression of $68 \%$ for ASO 1 and $44 \%$ for ASO 2.

\section{ASOs Reduce Kras Expression in Vivo}

Oligonucleotides were administered subcutaneously to adult male Wistar rats on alternate days at a dose of $12.5 \mathrm{mg} / \mathrm{kg}$ for 6 days. Sequential sections of renal tissue were immunostained for aquaporin 1 to label proximal convoluted tubules, for aquaporin 2 to label distal convoluted tubules and collecting ducts, and with an anti-oligonucleotide antibody to localize oligonucleotide deposition. Figure 2A illustrates that oligonucleotide is deposited in proximal convoluted tubules only, with no staining apparent in either the glomeruli or distal convoluted tubules and collecting ducts. RTqPCR analysis of renal tissue demonstrated oligonucleotide effects reflecting those seen in vitro. No significant difference was seen between $\mathrm{CO} 2-$ and vehicle-only-treated groups regarding Kras expression. However, $12 \%$ and $66 \%$ knockdown of Kras mRNA expression was seen in the ASO 1 and ASO 2

A

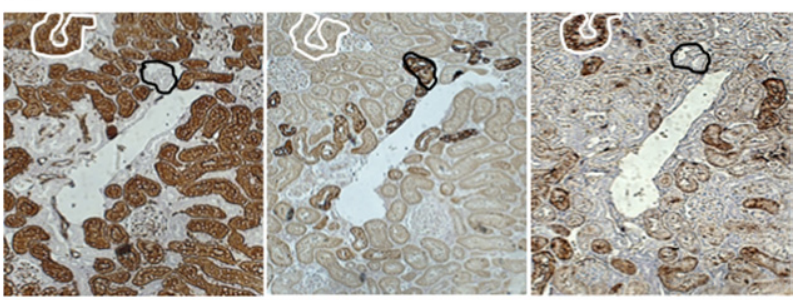

Aquaporin 1

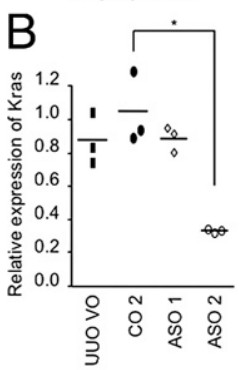

Aquaporin 2

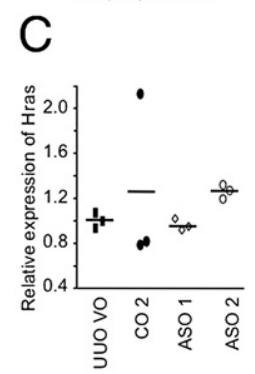

Oligonucleotide
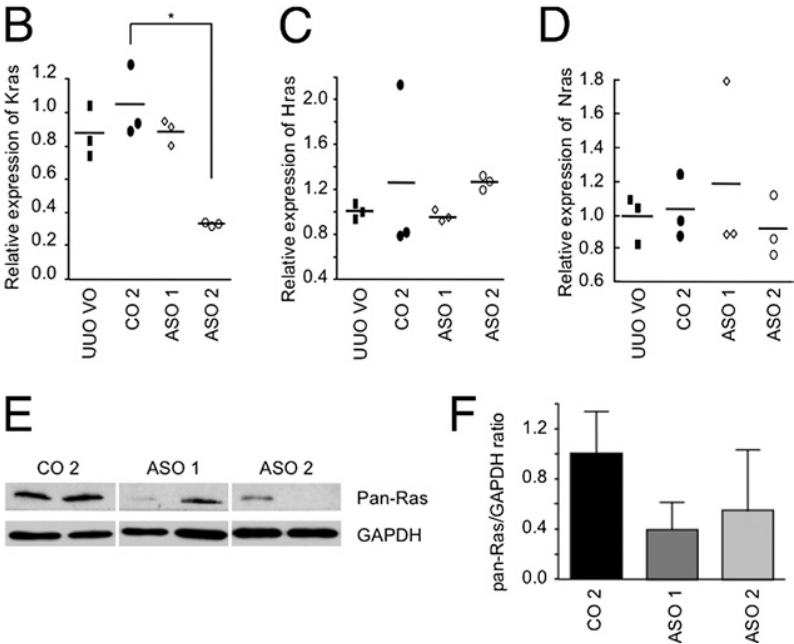

Figure 2. ASOs reduce Kras expression in vivo. Control or antisense oligonucleotides were administered to Wistar rats subcutaneously on alternate days at a dose of $12.5 \mathrm{mg} / \mathrm{kg}$ over 6 days (three doses). A: Sequential sections were obtained from a single rat kidney and were immunostained for aquaporin 1 , aquaporin 2 , and oligonucleotide deposits as labeled. The corresponding proximal convoluted tubule in each section is outlined in white, and the corresponding distal convoluted tubule and collecting duct in each section is outlined in black. mRNA and total protein were extracted from whole renal tissue after sacrifice. B-D: mRNA was subjected to RT-qPCR for Kras, Hras, and Nras as indicated ( $n=3$ animals). E and F: Pan-Ras was immunoprecipitated from a $0.5-\mathrm{mg}$ aliquot of all protein extracted from whole kidney tissue. The precipitate was then subjected to Western blot analysis for total pan-Ras. To demonstrate equality of protein content among samples studied, glyceraldehyde-3-phosphate dehydrogenase (GAPDH) also was quantitated, by Western blot analysis, in cell lysates after pan-Ras immunoprecipitation. The blot is representative of three similar experiments. Error bars are SEM. ${ }^{*} P<0.001$. VO, vehicle only 
groups, respectively. No significant difference for any group was seen regarding Hras and Nras expression (Figure 2, B-D). Although modest effects were seen in Kras mRNA expression after ASO treatment, these were translated into decreases in total Ras protein expression by $60 \%$ in the ASO $1-$ and $44 \%$ in the ASO 2-treated groups compared with the $\mathrm{CO} 2$ control group (Figure 2, E and F).

\section{Kras and Nras Expression Is Increased in UUO}

UUO was caused in male Wistar rats as described previously herein. Renal tissue from obstructed kidneys, contralateral nonobstructed kidneys, and the kidneys of sham-operated animals was analyzed for Ras mRNA levels. This analysis demonstrated increases in Kras and Nras expression by $70 \%$ and $80 \%$, respectively, in obstructed kidneys compared with in the contralateral kidneys or kidneys from sham-operated rats (Figure 3). No significant difference in Hras expression among groups was found.

\section{Kras ASOs Reduce Kras mRNA Expression in UUO}

Administration of Kras ASOs greatly reduced Kras expression in obstructed kidneys in a rat model of UUO compared with renal tissue retrieved from UUO vehicleonly and UUO control oligonucleotide-treated groups. ASO 1 reduced Kras mRNA levels by $61 \%$ and $56 \%$ compared with the scrambled oligonucleotides SO 1 and SO 2, respectively, whereas ASO 2 reduced expression by $97 \%$ compared with both these scrambled controls $(P<0.01)$. No statistically significant change in either $N$ or Hras mRNA levels was found in either ASO group. Administration of SO 1 or SO 2 resulted in a trend toward increased expression of all isoforms of ras, but only the rise in Nras in the SO 1-treated group was statistically significant $(P<0.01)$ (Figure 4). Administration of Kras ASOs did not affect gross phenotypic appearance or behavior of the animals or have a significant effect on growth or weight gain (see Supplemental Figure S1 at http://ajp.amjpathol.org).

\section{Kras ASOs Inhibit Fibrosis in UUO}

Sixteen days of UUO resulted in macroscopic, histologic, and molecular evidence of obstructive nephropathy and fibrosis. Compared with renal tissue from sham-operated animals, obstructed kidneys had dilated tubules, flattened tubular epithelial cells, greatly increased interstitial volumes, and prominent inflammatory infiltrates. Fibrosis was markedly increased, with evidence of substantial myofibroblastic and macrophage activity as reflected by an increase in $\alpha$-SMA expression and ED-1-positive cells (Figures 5 and 6). The degree of interstitial fibrosis was determined by staining with PMT and PSR, and collagen I deposition was stained directly using specific antibodies (Figure 5A). ASOs were scored against CO 2 and SO 1 or SO 2 by a consultant histopathologist blinded to

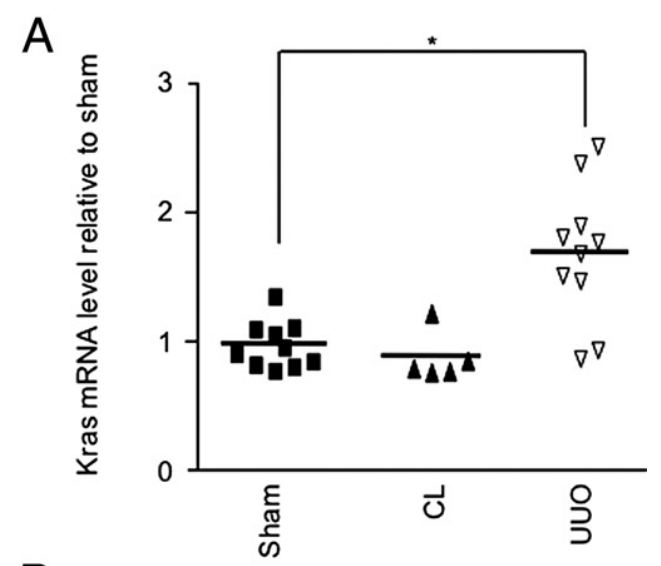

B

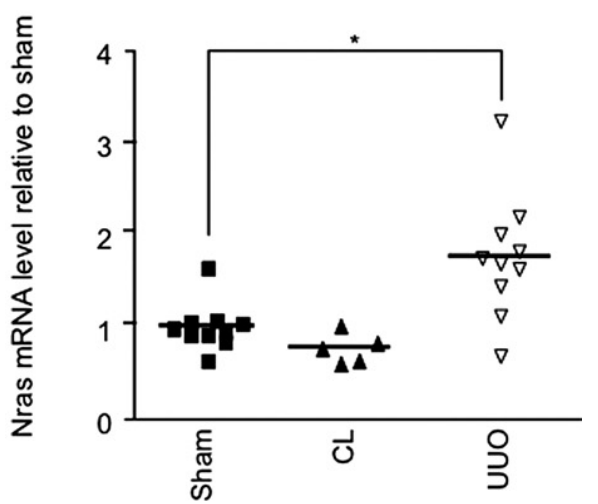

C

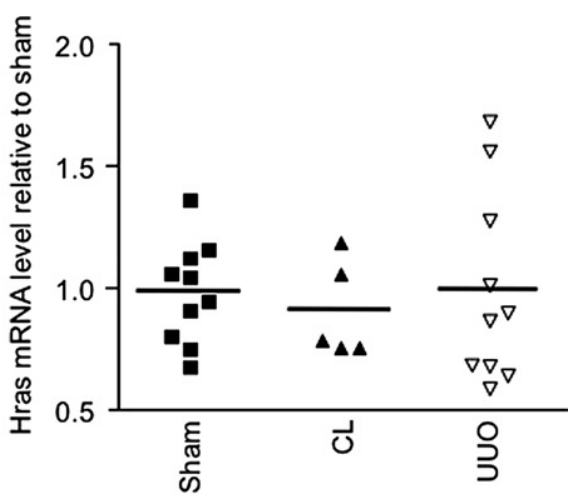

Figure 3. Kras expression is increased in UUO. UUO was caused in male Wistar rats. mRNA was extracted from renal tissue from obstructed kidneys, contralateral kidneys (CL), nonobstructed kidneys, and kidneys from sham-operated animals. Each sample ( $n=5$ to 10) underwent RTqPCR for Kras, Hras, and Nras. Expression of each gene was compared; glyceraldehyde-3-phosphate dehydrogenase served as an internal control. ${ }^{*} P<0.001$.

treatment. Percentage fibrosis scores as derived from PMT- and PSR-stained sections were averaged to provide an overall mean percentage fibrosis score (Figure $5 \mathrm{~B})$. This demonstrated an increase in the interstitial fibrosis score in vehicle-only-treated UUO models to $61.9 \%$ compared with the control sham-operated kidney group baseline fibrosis score of $5.8 \%$. No significant difference in fibrosis was observed after administration of CO 2, with a mean observed fibrosis score of $67 \%$ in this group. Although the administration of SO 1 and SO 2 reduced fibrosis scores to $40.1 \%$ and $36.6 \%$, respec- 
A

B
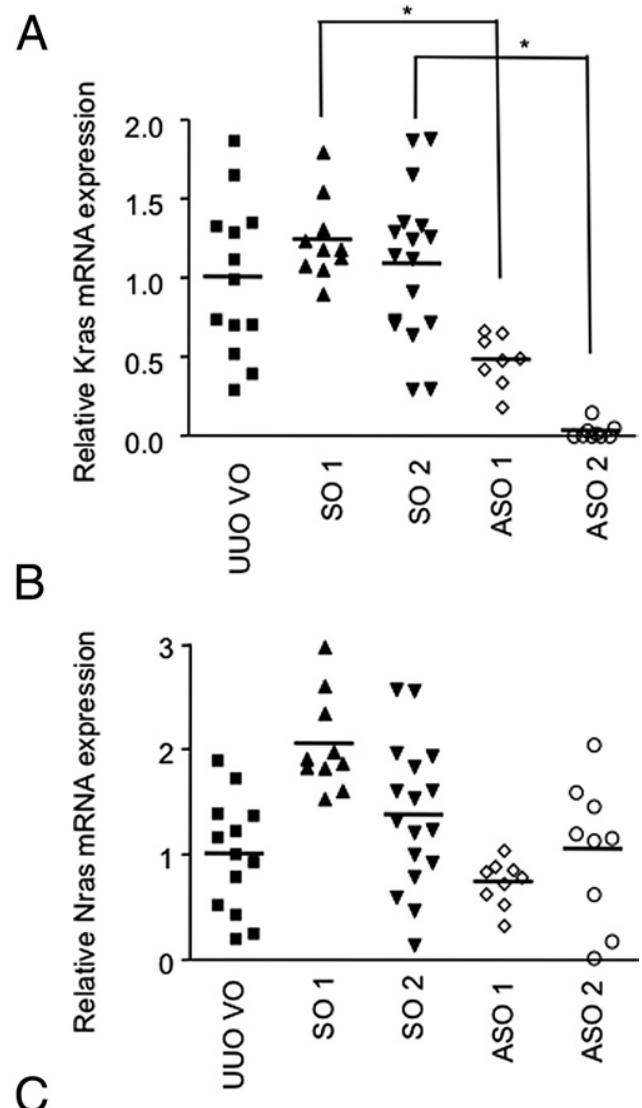

C

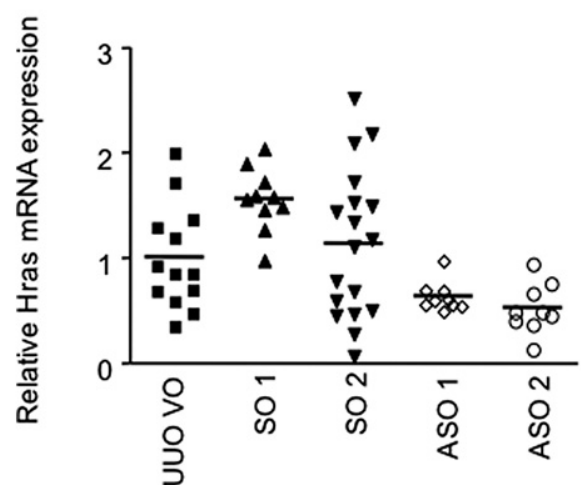

Figure 4. Kras ASOs reduce Kras mRNA levels in UUO. After 16 days of UUO, mRNA was extracted from whole renal tissue from obstructed kidneys and subjected to RT-qPCR for $\operatorname{Kras}(\mathbf{A}), \operatorname{Nras}(\mathbf{B})$, and Hras (C). $n=10$ to 13 animals; each result is a mean of triplicate measures. ${ }^{*} P<0.001$. VO, vehicle only.

tively, the administration of active Kras ASOs additionally reduced fibrosis, with ASO 1 reducing the fibrosis score to $17 \%(P<0.001)$ and $A$ SO 2 reducing it to $20.3 \%(P<$ $0.01)$.

Collagen I deposition was observed to increase from $9 \%$ in sham-operated kidneys to $50 \%$ in vehicle-treated obstructed kidneys. Administration of $\mathrm{CO} 2$ did not significantly affect this finding (collagen I deposition score, $40 \%$ ). However, administration of ASO 1 and ASO 2 reduced the collagen deposition score to $18.4 \%$ and $17 \%$, respectively ( $P<0.05$ for both), compared with $\mathrm{CO} 2$ (Figure 5C).

\section{Kras ASOs Inhibit $\alpha$-SMA Expression While Increasing Inflammation in UUO}

$\alpha$-SMA is a well-recognized marker of myofibroblast activity. Figure 6A shows tissue sections stained for $\alpha$-SMA from sham and UUO models, with quantified results shown in Figure 6B. Expression of this protein rose from $1 \%$ to $44.5 \%$ of the total area after 16 days of UUO. This was not significantly altered by the administration of $\mathrm{CO} 2$ (53\%) but was reduced to $21 \%$ and $32 \%$ by SO 1 and SO 2 , respectively. Marked edema was also present. Administration of ASO 1 and ASO 2, however, further reduced the expression of $\alpha$-SMA to $3.9 \%$ and $20 \%$, respectively, in the presence of similar levels of edema. To quantify $\alpha$-SMA expression further, Western blot analysis for $\alpha$-SMA was performed on protein extracted from renal tissue. This demonstrated a large up-regulation in protein expression after UUO that was not affected by the administration of control oligonucleotides (Figure 6, C and D). However, ASO 1- and ASO 2-treated groups exhibited a marked decrease in $\alpha$-SMA expression that almost,

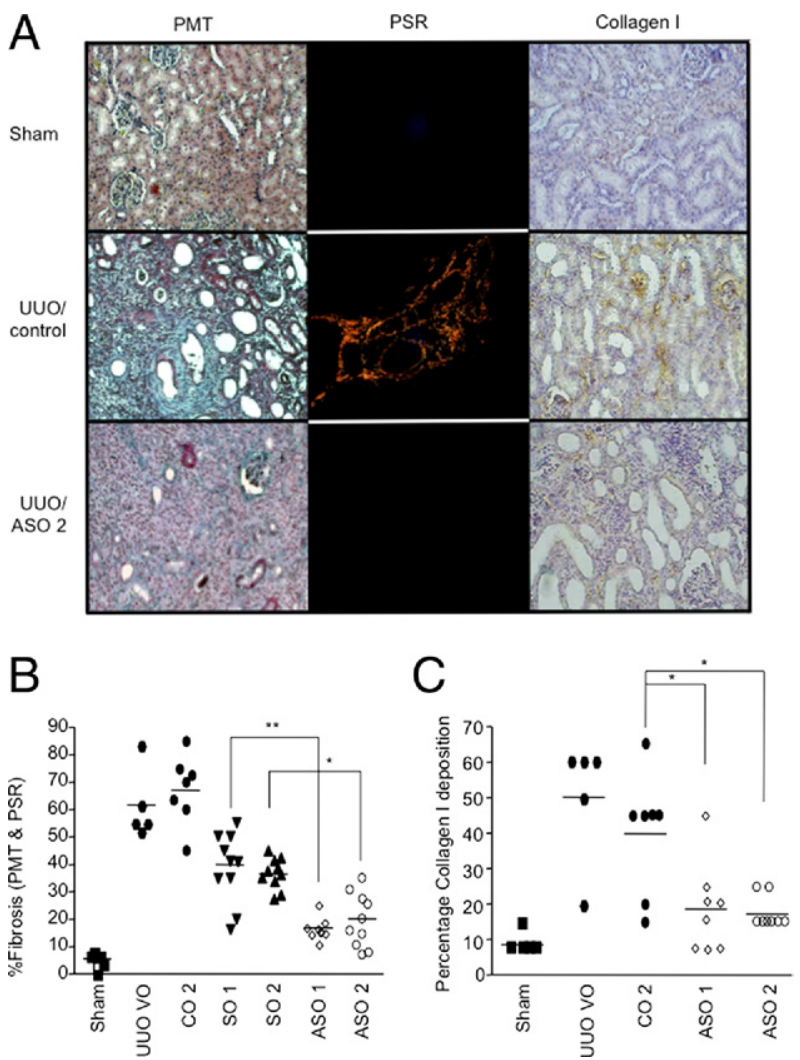

Figure 5. Kras ASOs inhibit fibrosis in UUO. A: Tissue sections were fixed and stained with PMT or PSR or were immunostained for collagen I as indicated. UUO/control indicates SO 2 oligonucleotide for PMT and PSR and CO 2 for collagen I. The PSR sections were viewed under cross-polarized light. Images shown are representative. Original magnification, $\times 100$ for all images. B: Each animal was assigned a mean fibrosis score by averaging the percentage of fibrosis observed with PMT and the percentage of fibrosis observed with PSR. This score is plotted against the treatment the animal received as indicated. C: For each animal, the percentage of collagen deposition was scored by a consultant histopathologist as described in Materials and Methods and is plotted against the treatment the animal received as indicated. $n=5$ to 10 animals. ${ }^{*} P<0.05$. VO, vehicle only. 


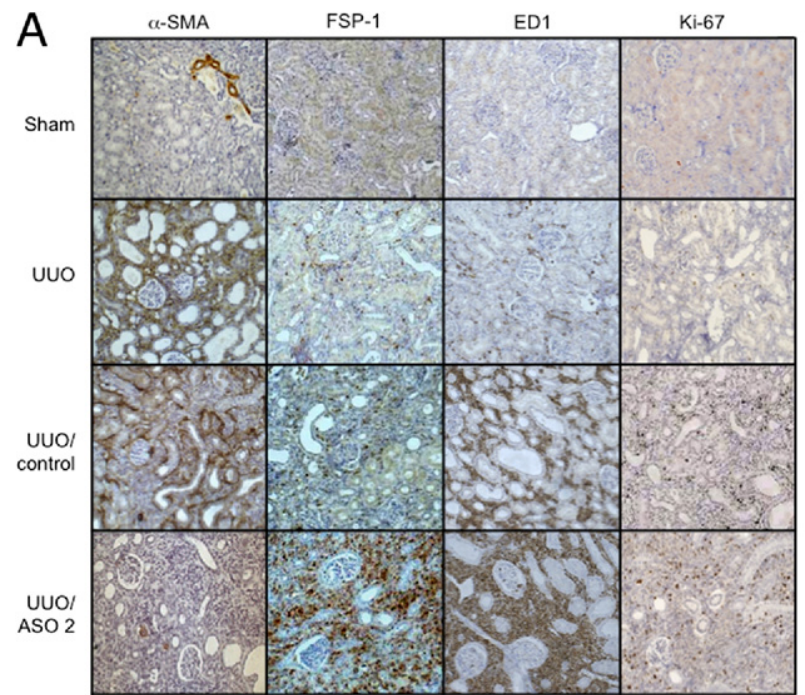

$\mathrm{B}_{\text {s }}$

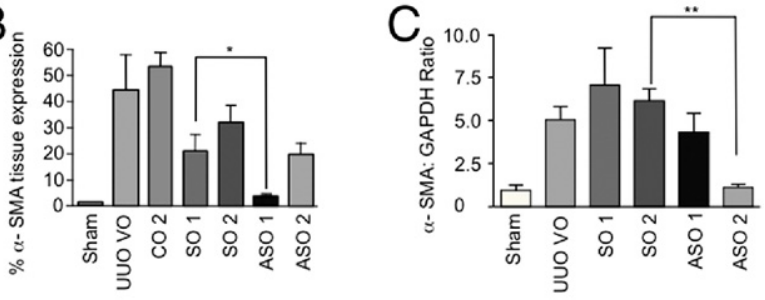

D

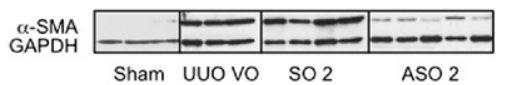

Figure 6. Kras ASOs inhibit $\alpha$-SMA expression despite increased interstitial cell numbers in UUO. Oligonucleotides were administered subcutaneously to adult male Wistar rats on alternate days at a dose of $12.5 \mathrm{mg} / \mathrm{kg}$ across 16 days after UUO or a sham operation. The animals were then sacrificed $\mathbf{A}$ : Tissue sections were fixed and immunostained for $\alpha$-SMA, FSP-1, ED-1, or $\mathrm{Ki}-67$ as indicated. Images shown are representative. Original magnification, $\times 100$ for all images. B: Five random cortical views per section were scored in a blinded manner by measuring staining intensity and area using Nikon NIS-Elements (basic research) software. The results are plotted as percentage of $\alpha$-SMA tissue expression against the individual treatments as indicated. $n$ $=5$ to 10 animals. $\mathbf{C}$ : Total protein was extracted from whole renal tissue and underwent Western blot analysis for $\alpha$-SMA. D: Densitometry was performed, and $\alpha$-SMA/glyceraldehyde-3-phosphate dehydrogenase (GAPDH) density ratios are plotted against each treatment as indicated. $n=3$ to 5 animals. Error bars are SEM. ${ }^{*} P<0.05,{ }^{* *} P<0.001$. VO, vehicle only.

in the latter group, reverted to basal levels (as expressed in sham-operated animals).

Renal tissue from sham-operated and obstructed kidneys was also stained for FSP-1 expression (Figure 6A). The discrete nature of staining allowed for stained cells to be categorized as glomerular, tubular, or interstitial. As expected, staining for FSP-1 was minimal in all compartments in sham-operated kidneys. Obstruction led to a nonsignificant rise in tubular expression of FSP-1 (data not shown) and to an increase in interstitial expression from 4 FSP-1-positive cells per view to 28 FSP-1-positive cells per view $(P<0.05)$ (Figure $7 \mathrm{~A})$. Administration of oligonucleotides, either control or ASO, did not significantly alter FSP-1 expression in either glomerular or tubular compartments compared with the vehicle-only group (data not shown). Although interstitial expression of FSP-1 increased on administration of scrambled oligonucleotides and Kras ASOs compared with administra- tion of vehicle-only UUO, the increase was significant only on administration of ASO $1(P<0.001)$.

Measurement of intensity and area of ED-1 antibody staining (against the macrophage marker CD 68) using Nikon NIS-Elements (basic research) software clarified the nature of the increased interstitial infiltrate (Figures 6 and 7B). Sham-operated samples revealed few macrophages per view $(<1 \%$ of the total area). As expected,
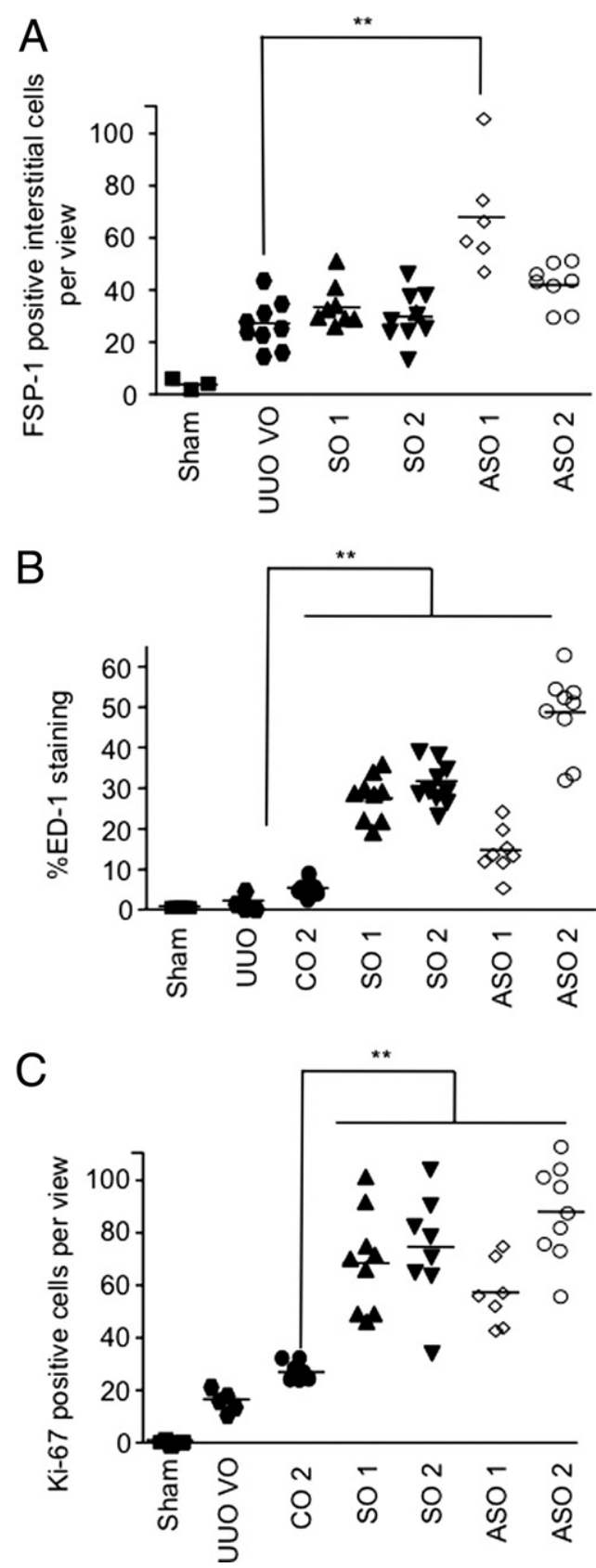

Figure 7. ASOs increase inflammation in UUO. Tissue sections were immunostained for FSP-1 (A), ED-1 (B), and Ki-67 (C). For ED-1 staining, five random cortical views per section were scored in a blinded manner by measurement of staining intensity and area using Nikon NIS-Elements (basic research) software. The results are plotted as percentage of ED-1 tissue expression. For Ki-67 and FSP-1 staining, cells that marked were counted by two people in a blinded manner (light microscopy; original magnification, $\times 200$ ) in 10 random fields of cortex. $n=5$ to 10 animals. ${ }^{* *} P<0.001$. VO, vehicle only. 
obstruction resulted in an increase in ED-1 staining to $2.4 \%$, reflecting the inflammatory infiltration associated with $\cup \cup O$. The administration of $\mathrm{CO} 2$ led to a doubling of ED-1 staining (5.5\%). However, ED-1 staining further increased significantly with the administration of scrambled and antisense oligonucleotides. SO 1 and SO 2 administration increased ED-1 staining to 28\% and $32 \%$, respectively $(P<0.001)$. Administration of ASO 1 increased ED-1 staining to $15 \%$, but administration of ASO 2 resulted in a more substantial increase to $49 \%(P<0.001)$.

Ras is known to play a central role in cell proliferation. Our previous work has demonstrated that Kras knockdown reduces stimulated proliferation in renal fibroblasts. ${ }^{18,19}$ Kidney sections were, therefore, stained for Ki-67, a marker of actively dividing cells. In the interstitial compartment, Ki-67 expression was increased by 2.5fold to fourfold in the SO 1-, SO 2-, ASO 1-, and ASO 2-treated groups compared with the $\mathrm{CO} 2$-treated group $(P<0.01)$ (Figures 6 and $7 C$ ).

\section{Oligonucleotides also Target Other Tissues}

Two hours after systemic administration, the highest concentrations of oligonucleotides are found in the liver and kidneys. ${ }^{21}$ RT-qPCR analysis of hepatic tissue produced results comparable with those obtained from the kidney. Both SO 1 and SO 2 administration resulted in an increase in Kras hepatic mRNA levels compared with the vehicle-only-treated UUO group. Administration of ASO 1 and ASO 2 reduced Kras hepatic expression by $67 \%$ and $82 \%$, respectively, compared with their paired, basematched scrambled oligonucleotides (see Supplemental Figure S2B at http://ajp.amjpathol.org). Oligonucleotide deposits could also be detected in other tissues, including heart and muscle, although less than in liver and kidney (see Supplemental Figure S2A at http:// ajp.amjpathol.org). Despite this widespread uptake of oligonucleotide, wound healing did not differ, either clinically or histologically, between vehicle-treated animals and those treated with ASOs (see Supplemental Figure S2C at $h$ ttp://ajp.amjpathol.org).

In H\&E-stained hepatic tissue, we found mild to moderate inflammation in both lobule and portal tract on administration of oligonucleotide, with ASO 2 causing more inflammation than ASO 1. Architecture was normal (see Supplemental Figure S3A at http://ajp.amjpathol.org). Similarly, mild inflammation was noted in the heart tissue stained for ED-1 from oligonucleotide-treated animals, with ASO 2 being the most inflammatory oligonucleotide (see Supplemental Figure S3, B and C, at http://ajp.amjpathol.org).

\section{Discussion}

In this study, we demonstrated that a single isoform of ras can be specifically targeted in vitro and in vivo using ASOs. Ras is a key signaling molecule pivotal to control of cell growth and differentiation in many cell types. Our previous work highlighted Kras as the predominant iso- form in renal fibroblasts. This isoform is, therefore, a desirable target to maximize the effect on the kidney while minimizing other tissue involvement. Binding of the ASO to target mRNA results not only in steric inhibition of translation by the ribosomal complex but also, and more important, in the induction of RNase $\mathrm{H}$, which cleaves the $3^{\prime}$-O-P-bond of the RNA molecule. This mechanism of action theoretically provides 100\% specificity for the target gene, an unachievable goal for most conventional pharmacologic agents. In addition, parenterally administered ASO accumulates principally in the kidneys, with a kidney/plasma concentration ratio of 80:1 after 2 hours. ${ }^{21}$

Although we and other researchers have been investigating the role of Ras in renal fibrosis for some years, Ras has become a more pertinent target recently. RASAL1, a Ras GTPase activating protein and, hence, a Ras inhibitor, has been found to be down-regulated in fibroblasts from fibrotic kidneys and not in fibroblasts from nonfibrotic kidneys as a result of hypermethylation of its parent gene. Hypermethylation results in reduced RASAL1 gene transcription and, hence, in Ras activation. ${ }^{15}$ This phenomenon is also present in colonic neoplasms, where decreased RASAL1 expression was noted in more advanced tumors expressing wild-type Kras. ${ }^{22}$

In this study, we demonstrated that parenteral administration of ASOs targeting Kras can significantly inhibit the onset of fibrosis in a model of UUO as measured by PMT, PSR, collagen I deposition, and $\alpha$-SMA expression. This inhibition is achieved despite an increase in the observed interstitial inflammation demonstrated in the kidneys of treated animals. This inflammation is not specific to the Kras ASOs as it seems to be the same for the scrambled control oligonucleotides. The nonstoichiometric control oligonucleotide (CO 2) caused significantly less inflammation, suggesting that this phenomenon is probably not sequence related but instead depends on relative nucleotide compositions of each oligonucleotide. We also stained the samples for FSP-1 as an assumed marker for interstitial fibroblasts. Although expression of FSP-1 increased with UUO, unlike $\alpha$-SMA, it increased further in scrambled oligonucleotide-treated and ASOtreated kidneys. FSP-1 is expressed by interstitial fibroblasts, but it is also expressed by inflammatory cells. This expression probably overwhelmed any reduction in fibroblast numbers in this study. ${ }^{23}$ Although we did not specifically characterize the nature of the inflammatory reaction, we did demonstrate that it contained both macrophages and actively dividing cells likely not to be of the macrophage inflammatory lineage. Whether these inflammatory cells or increased numbers of interstitial fibroblasts cause increased Kras and Nras expression in UUO is unclear. Administration of the Kras ASOs did, however, reduce total kidney Kras expression well below values seen in UUO alone, despite increased inflammation in the treated groups.

Although the kidneys are preferentially targeted by small oligonucleotides, they also deposit in other tissues throughout the body, including the liver and, to a lesser degree, the heart and striated muscle. We noted a moderate reduction in Kras expression in the livers of ASOtreated animals and an associated mild inflammation in 
the liver and heart, suggesting a widespread oligonucleotide-related inflammatory reaction. This caused no significant architectural disturbance or evidence of fibrosis. Neither did any treated animal exhibit outward signs of ill health, gaining weight at the same rate as untreated animals with UUO.

In conclusion, we demonstrated that targeting Kras with ASOs can prevent the onset of fibrosis in a rat model of unilateral obstruction, even in the presence of significant inflammation. New ASO design techniques promise to produce noninflammatory ASOs, which may be used in future studies.

Chronic inflammation is generally thought, in clinical medicine, to inevitably lead to irreversible fibrosis. The dissociation between inflammation and fibrosis demonstrated herein suggests that this may not always be so. If fibrosis can be inhibited while the initial insult resulting in inflammation is either removed or controlled, irreversible scarring of the kidney may be avoided. Although further study is called for, we believe that ASOs targeting Kras represent a first step in developing a true "antifibrotic" therapy, one that in the future may prevent renal fibrosis.

\section{Acknowledgments}

We thank the late Dr. Neelanjana Dutt, who contributed substantially to this work with her expertise in renal histopathology.

\section{References}

1. Norman JT, Fine LG: Progressive renal disease: fibroblasts, extracellular matrix, and integrins. Exp Nephrol 1999, 7:167-177

2. Iwano M, Plieth D, Danoff TM, Xue C, Okada H, Neilson EG: Evidence that fibroblasts derive from epithelium during tissue fibrosis. J Clin Invest 2002, 110:341-350

3. Lin SL, Kisseleva T, Brenner DA, Duffield JS: Pericytes and perivascular fibroblasts are the primary source of collagen-producing cells in obstructive fibrosis of the kidney. Am J Pathol 2008, 173:1617-1627

4. Eddy AA: Molecular basis of renal fibrosis. Pediatr Nephrol 2000, 15:290-301

5. Wittinghofer A, Scheffzek K, Ahmadian MR: The interaction of Ras with GTPase-activating proteins. FEBS Lett 1997, 410:63-67

6. Bos JL: ras oncogenes in human cancer: a review. Cancer Res 1989, 49:4682-4689

7. Clarke HC, Kocher HM, Khwaja A, Kloog Y, Cook HT, Hendry BM: Ras antagonist farnesylthiosalicylic acid (FTS) reduces glomerular cellular proliferation and macrophage number in rat thy-1 nephritis. J Am Soc Nephrol 2003, 14:848-854
8. Dockrell ME, Phanish MK, Hendry BM: Tgf- $\beta$ auto-induction and connective tissue growth factor expression in human renal tubule epithelial cells requires N-ras. Nephron Exp Nephrol 2009, 112:e71e79

9. Grande MT, Fuentes-Calvo I, Arevalo M, Heredia F, Santos E, Martinez-Salgado C, Rodriguez-Puyol D, Nieto MA, Lopez-Novoa JM: Deletion of $\mathrm{H}$-Ras decreases renal fibrosis and myofibroblast activation following ureteral obstruction in mice. Kidney Int 2010, 77:509-518

10. Janda E, Lehmann K, Killisch I, Jechlinger M, Herzig M, Downward J, Beug $H$, Grunert S: Ras and TGF $\beta$ cooperatively regulate epithelial cell plasticity and metastasis: dissection of Ras signaling pathways. J Cell Biol 2002, 156:299-313

11. Kocher HM, Moorhead J, Sharpe CC, Dockrell ME, AI-Nawab M, Hendry BM: Expression of Ras GTPases in normal kidney and in glomerulonephritis. Nephrol Dial Transplant 2003, 18:2284-2292

12. Lahsnig C, Mikula M, Petz M, Zulehner G, Schneller D, van Zijl F, Huber H, Csiszar A, Beug H, Mikulits W: ILEI requires oncogenic Ras for the epithelial to mesenchymal transition of hepatocytes and liver carcinoma progression. Oncogene 2009, 28:638-650

13. Martinez-Salgado C, Fuentes-Calvo I, Garcia-Cenador B, Santos E, Lopez-Novoa JM: Involvement of $\mathrm{H}$ - and $\mathrm{N}$-Ras isoforms in transforming growth factor- $\beta 1$-induced proliferation and in collagen and fibronectin synthesis. Exp Cell Res 2006, 312:2093-2106

14. Rodriguez-Pena AB, Grande MT, Eleno N, Arevalo M, Guerrero C, Santos E, Lopez-Novoa JM: Activation of Erk1/2 and Akt following unilateral ureteral obstruction. Kidney Int 2008, 74:196-209

15. Bechtel W, McGoohan S, Zeisberg EM, Muller GA, Kalbacher $H$, Salant DJ, Muller CA, Kalluri R, Zeisberg M: Methylation determines fibroblast activation and fibrogenesis in the kidney. Nat Med 2010, 16:544-550

16. Johnson L, Greenbaum D, Cichowski K, Mercer K, Murphy E, Schmitt E, Bronson RT, Umanoff H, Edelmann W, Kucherlapati R, Jacks T: $\mathrm{K}$-ras is an essential gene in the mouse with partial functional overlap with N-ras. Genes Dev 1997, 11:2468-2481

17. Santos E, Nebreda AR: Structural and functional properties of ras proteins. FASEB J 1989, 3:2151-2163

18. Sharpe CC, Dockrell ME, Noor MI, Monia BP, Hendry BM: Role of Ras isoforms in the stimulated proliferation of human renal fibroblasts in primary culture. J Am Soc Nephrol 2000, 11:1600-1606

19. Sharpe CC, Dockrell ME, Scott R, Noor MI, Cowsert LM, Monia BP, Hendry BM: Evidence of a role for Ki-Ras in the stimulated proliferation of renal fibroblasts. J Am Soc Nephrol 1999, 10:1186-1192

20. Wang JH, Hendry BM, Sharpe CC: Silencing genes in the kidney: antisense or RNA interference? Nephrol Dial Transplant 2008, 23 : 2115-2118

21. Sands H, Gorey-Feret LJ, Cocuzza AJ, Hobbs FW, Chidester D, Trainor GL: Biodistribution and metabolism of internally $3 \mathrm{H}$-labeled oligonucleotides, I: comparison of a phosphodiester and a phosphorothioate. Mol Pharmacol 1994, 45:932-943

22. Ohta M, Seto M, ljichi H, Miyabayashi K, Kudo Y, Mohri D, Asaoka Y, Tada M, Tanaka Y, Ikenoue T, Kanai F, Kawabe T, Omata M: Decreased expression of the RAS-GTPase activating protein RASAL1 is associated with colorectal tumor progression. Gastroenterology 2009, 136:206-216

23. Le Hir M, Hegyi I, Cueni-Loffing D, Loffing J, Kaissling B: Characterization of renal interstitial fibroblast-specific protein 1/S100A4-positive cells in healthy and inflamed rodent kidneys. Histochem Cell Biol 2005, 123:335-346 